\title{
Bessere Oberflächenqualität dank geschäumter Klebstoffe
}

$\mathrm{H}^{\mathrm{s}}$ .B. Fuller hat mit Rakoll 4330 eine neue Generation wasserbasierter Klebstoffe eingeführt, die schäumbar sind und den stetig steigenden Anforderungen der holzverarbeitenden Indust-

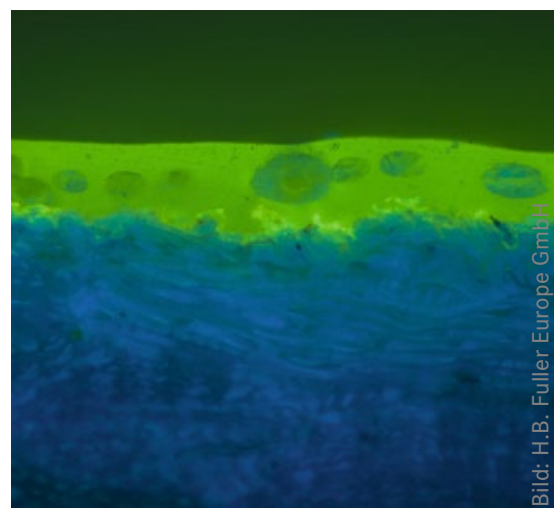

Bei Verwendung schäumbarer Klebstoffe wird das Ausmaß der Faserschwellung minimiert rie nach besserer Oberflächenqualität gerecht werden.

Aufgetragen als homogener und stabiler Mikroschaum, vermindert dieser einkomponentige PVAc-Klebstoff das Aufquellen von Fasern und das schnelle Penetrieren von Wasser in die Zellen, was zu einer höheren Oberflächenqualität führt. Zum Applizieren dieses neuen Klebstoffs dient ein spezielles Schaumequipment, das von H.B. Fuller in Zusammenarbeit mit Geräteherstellern wie Heitec-MST entwickelt wurde.

Dazu Walter Stadlbauer, Technischer Leiter für den Bereich Holzklebstoffe bei H.B. Fuller in Europa: „Die Entwicklung von optimalen Lösungen für unsere Kunden ist eines unserer Hauptziele. Die Oberflächenqualität ist eine komplexe Thematik und hat seit Jahren hohe Priorität bei unseren Kunden und Maschinen- herstellern. Dank dieser neuen Technologie können wir unseren Kunden helfen, durch Verwendung von schäumbaren Klebstoffen eine noch bessere Oberfläche zu erreichen. Das Wasser wird dabei nicht sofort von den Fasern aufgenommen, was das Ausmaß der Faserschwellung minimiert und den Effekt einer glatteren Oberfläche hat.“

Der neue Klebstoff bietet neben der besseren Benetzung der Oberflächen eine längere offene Zeit und eine reduzierte Auftragsmenge gegenüber alternativen, nicht schäumbaren Klebstoffen auf Harnstoff-Formaldehyd-Basis. Er erfüllt die Anforderungen nach DIN EN 204: D3 und ist vom ift Rosenheim zertifiziert.

Weitere Infos: H.B. Fuller Europe GmbH, www.hbfuller.com

\section{Vergussmassen für Elektronik im Unterwasserbereich}

E lektronik im Unterwasserbereich zuverlässig zu schützen, stellt eine hohe Anforderung an die Leistungsfähigkeit von Vergussmassen dar. Die zum Beispiel für den Verguss von Unterwasserkameras, Tauchlampen oder Mess-

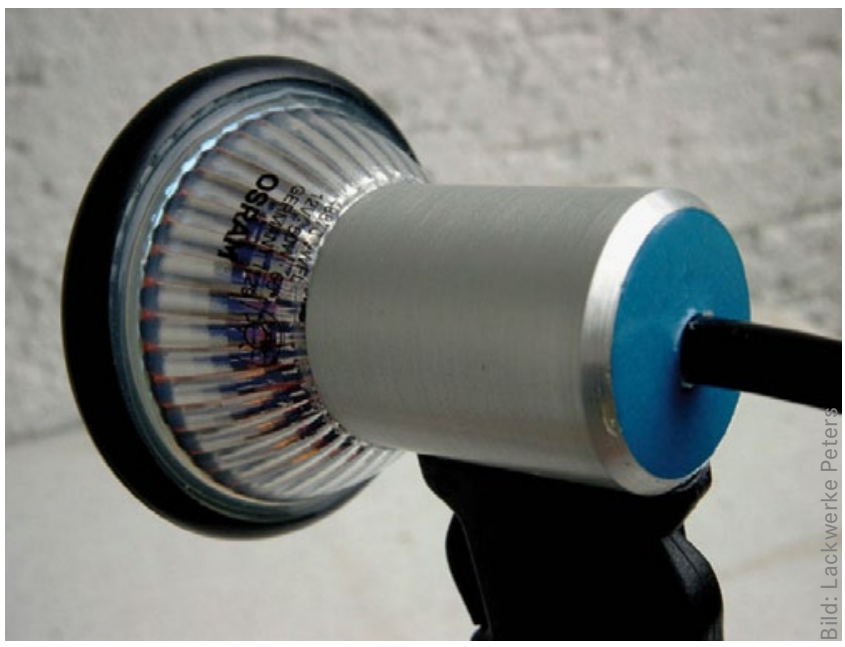

Das zum Beispiel für diese Tauchlampe eingesetzten Vergussmaterial muss hohe Anforderungen erfüllen. sensoren eingesetzten Materialien müssen nicht nur die elektronischen Isolationseigenschaften erfüllen, sondern sich auch durch eine außergewöhnlich hohe Wasser- und Hydrolysebeständigkeit sowie hohe mechanische Festigkeit und

Widerstandsfähigkeit auszeichnen.

Solchen Einsätzen gewachsen sind zwei von den Lackwerken Peters angebotene 2-Komponenten-Vergussmassen auf Polyurethanharzbasis, die blaue Wepuran-Vergussmasse VU 4453/101 $\mathrm{WR}($ Index WR = wasser-resistent) und das farblose, kristallklare Wepuran-Gießharz VT 3402 KK-NV.

Während der Verguss mit der blauen Wepuran-Vergussmasse völlig undurchsichtig ist, können mit dem farblosen, kristallklaren Wepuran-Gießharz auch lichtoptische Bauelemente gekapselt werden.

Aufgrund der hohen Elastizität werden bei Einsatz beider Vergussmassen Materialspannungen bei Druckschwankungen und Temperaturwechseln reduziert, sodass der wasserdichte Verguss erhalten bleibt. Speziell für Anwendungen mit höchsten Anforderungen an optische Eigenschaften sowie in der Sensortechnik empfiehlt sich der Einsatz der farblosen Variante, die sich durch eine extrem hohe Transparenz selbst bei Bewitterung und UV-Einstrahlung auszeichnet.

Weitere Infos: Lackwerke Peters GmbH + Co KG, 47882 Kempen, peters@peters.de,www. peters.de 